\title{
Research Use and Evidence-Based Practice Among Swedish Medical Social Workers: A Qualitative Study
}

\author{
Camilla Udo $^{1} \cdot$ Henrietta Forsman ${ }^{1} \cdot$ Marcus Jensfelt ${ }^{2} \cdot$ Maria Flink $^{2,3}$ (i)
}

Published online: 29 January 2018

(c) The Author(s) 2018. This article is an open access publication

\begin{abstract}
The aim of this study was to explore medical social workers' perceptions of evidencebased practice (EBP), including factors relevant for the successful implementation of evidence into medical social work practice. Eight focus group interviews were conducted that included 27 medical social workers. Data were analyzed using qualitative content analysis, which resulted in two categories: "knowledge in practice" and "challenges in relation to the implementation of EBP" and four subcategories: "practice based on research evidence or experience", "obtaining new evidence of practice", "research and the social work context", and "barriers and facilitating factors". Participants tended to perceive EBP as theoretical and positivistic while perceiving their own knowledge as eclectic and experience-based. Although they perceived the relevance of research findings to their clinical practice, they expressed a need for support to translate research into policy and practice. They also reported that studies about their specific work were scarce. The medical social workers' suggestion for the facilitation of knowledge exchange needs further investigation.
\end{abstract}

Keywords Evidence-based practice $\cdot$ Focus group interviews $\cdot$ Knowledge $\cdot$ Medical social worker

\section{Introduction}

With the intention to do more good than harm, it is important to rely on medical practice that is rooted in evidencebased guidelines so that patients are given the exact care they need. Thus, from a patient safety perspective, the social worker needs to apply evidence-based practice (EBP).

Maria Flink

maria.flink@ki.se

Camilla Udo

cud@du.se

Henrietta Forsman

hfo@du.se

Marcus Jensfelt

marcus.jensfelt@karolinska.se

1 School of Education, Health and Social Studies, Dalarna University, Falun, Sweden

2 Function area of Social Work, Karolinska University Hospital, Solna, Sweden

3 Department of Learning, Informatics, Management and Ethics (LIME), Karolinska Institutet, Tomtebodavägen 18A, 17176 Stockholm, Sweden
One of the most cited conceptualizations of EBP in social work (Satterfield et al. 2009) is the three circle model: (1) the patient's state and circumstances, (2) the research evidence and the patient's preferences and (3) actions integrated with the professional's clinical expertise (Sacket et al. 1996; Haynes et al. 2002). Although there is considerable debate as to whether the concept of EBP, which is derived from a medical and positivistic context, is transferable to the complex context of social work (Morago 2006; Petr and Walter 2009), there has been increased interest in EBP in the field of social work (McNeece and Thyer 2004; Morago 2006; Rubin and Parrish 2007; Bergmark and Lundström 2011).

Applying EBP to everyday work is perceived by many as a paradigm shift compared to work that is based on previous knowledge and clinical expertise. It is important to consider the medical social workers' perspective of EBP, including their views of the factors that hinder or facilitate EBP. Numerous theories, models and frameworks have been used to illustrate central factors involved in the use of best available knowledge in practice. For example, the PARIHS (Promoting Action on Research Implementation in Health Services) framework (Rycroft-Malone 2004), now the iPARIHS framework (Harvey and Kitson 2015), has been widely used to guide intervention strategies and illustrate 
the interplay between the individuals involved in the EBP implementation, the innovation itself to be implemented and the context in which implementation occurs. Moreover, a number of individual characteristics of importance to the application of research evidence (such as problem-solving ability, attitudes and education) have been explored (Squires et al. 2011). In the current study, iPARIHS is used as a conceptual framework to help understand the results from the discussions of the different focus groups. According to the iPARIHS framework (Harvey and Kitson 2015), characteristics of the recipients (i.e. the users of evidence, their skills and knowledge, motivation, values and beliefs and power and authority) are critical factors for successful implementation into healthcare practices. The recipients' actions and reactions within the contextual setting are crucial for the successful implementation of EBP. Consequently, the perspective of the recipients plays a lead role in implementing EBP. Another core concept in the iPARIHS framework is the innovation itself (Harvey and Kitson 2015), i.e. the evidence to be implemented. Several characteristics of the innovation are relevant for successful implementation. Examples of such characteristics include underlying knowledge sources, compatibility with existing practices, values and usability. To achieve successful implementation, there is a need to include the practitioners (i.e. the medical social workers) early in the process. The third core concept in the iPARIHS is the context, conceptualized as the inner (local and organizational level) and outer (wider health system) context (Harvey and Kitson 2015). Among the contextual factors are formal, informal and senior management leadership and support. Other factors include the organizational culture, evaluation and feedback processes, organizational structure and systems and learning networks (Harvey and Kitson 2015).

Within medical social work, research on challenges related to EBP implementation is limited (Wike et al. 2014) and EBP is still a relatively new concept in social care (Manuel et al. 2009). The few studies that have specifically focused on medical social workers' attitudes show a low orientation towards EBP (Björkenheim 2007; Heiwe et al. 2013). Hence, in practice, EBP is still infrequently implemented within the field (Bellamy et al. 2006; Mullen and Bacon 2006; Rubin and Parrish 2007; Heiwe et al. 2013), even though some guiding models have recently been developed (Fugl-Meyer 2016). Because social work is conducted in a multitude of settings, each setting needs to be carefully studied to guide the successful implementation of EBP (Manuel et al. 2009). Social work conducted in healthcare settings is unique in many ways because of the context, therefore it is important to explore social work practice in these settings. Regardless of country, a medical social worker's main task concerns assessing the patient's different support needs for multidimensional health issues (e.g., traumas, disabilities and life-threatening illnesses), including a person's existential, social, emotional and environmental needs as well as collaborating within multidisciplinary teams in complex healthcare systems. The medical social worker is also responsible for conducting interventions for in- and outpatient care, as well as offering guidance to other care professionals based on an understanding of how trauma, disease or a chronic illness may affect the individual, his or her family and the social situation. The medical social worker often works on referral from a physician or a nurse, but sometimes the patients initiate contact. Although in the USA a typical medical social worker holds a Master of Social Work (MSW) degree, this is not the case in Sweden, where only a Bachelor of Social Work (BSW) degree is required.

EBP is an emerging approach among professionals in healthcare settings (Heiwe et al. 2011; Melnyk and Gallagher-Ford 2015). Learning more about the medical social workers' perceptions of EBP has the potential to contribute to an increased understanding of how best available knowledge can be implemented in medical social work settings to provide high quality and safe practice to patients. The aim of this study is to explore medical social workers' perceptions of EBP, including factors relevant for the successful implementation of evidence into medical social work practice.

\section{Methods}

\section{Design}

This is a qualitative study in which focus group interviews were conducted and analyzed by qualitative content analysis as inspired by Krippendorff (2013).

\section{Settings and Participants}

This study was conducted at six hospitals in two counties in Sweden in the winter 2015/2016. In the current study, 27 medical social workers ( 3 men and 24 women) were included in the study. All medical social workers had a BSW degree (equivalent to 3.5 years of university education). Two of the participants were MSW-trained medical social workers and five held a psychotherapist certificate in addition to their BSW degree. The participants were recruited using purposive sampling. Key persons (managers and contact persons) at each hospital received an e-mail, oral information, or both about the study along with a request to disseminate a call for participants among medical social workers with at least a BSW degree who had demonstrated experience working with counseling patients suffering from chronic conditions. All medical social workers who gave their consent to participate in the study and who were available during the data collection period were included. 


\section{Data Collection}

Eight focus group interviews (Morgan 1997) (3-4 participants per focus group) were conducted. Each interview followed a semi-structured interview guide (Kvale 2006). The interviews were constructed so that the participants could feel free to present their concerns and ideas related to the main theses of the study. During the interviews, questions were used that focused on the theories and methods used in the medical social workers' day-to-day practice, the way in which they obtained new knowledge, as well as their general perceptions of EBP. Three of the focus groups were led by two of the authors (MF and MJ) while the remaining five focus groups were led by the other authors (CU and HF). Three of the authors are experienced medical social workers with a MSW degree while the fourth author is an experienced registered nurse with a Master's degree in nursing; three of the authors are researchers with a $\mathrm{PhD}$ degree. Finally, two of the authors are experienced interviewers in qualitative research. The interviews, lasting between 60 and $75 \mathrm{~min}$, were tape recorded and transcribed verbatim.

\section{Data Analysis}

Data were analyzed by performing a qualitative content analysis as inspired by Krippendorff (2013). During the analysis, the findings were continuously discussed among the authors. The interviews were first compiled as one text and read several times by the first (CU) and last author (MF) to obtain a first impression before meaning units related to the aim of the study were identified by $\mathrm{CU}$ and discussed with MF. The meaning units were then condensed and labelled with codes by CU and MF. The codes were kept close to the participants' original descriptions and checked back with the meaning units several times before being compared and grouped based on similarities. The groups of codes were then used to form preliminary subcategories and categories. This process was led by $\mathrm{CU}$ and MF, although each step in the analysis was continuously discussed among all authors to make the analysis transparent. This process led to a slight modification of the categories before consensus was reached.

\section{Ethical Considerations}

Once the Regional Ethical boards in Stockholm and Uppsala had assessed the study and permission to proceed had been granted by the social workers' supervisors, the prospective participants were informed both verbally and in writing that participation was voluntary and that no data would be used to identify the participants individually. The anonymity was assured by combining the texts from different groups into a single text before conducting the analysis, using no quotations that would make it possible to identify the participants. Before obtaining each participant's written consent to take part in the study, confidentiality was ensured and they were informed that they could withdraw at any time during the study without consequences and without reporting a reason for their withdrawal.

\section{Results}

The study participants ranged in age from 26 to 62 $($ mean $=45)$ years. Each participant had a BSW degree and had worked as a medical social worker from 1 to 25 $($ mean $=9)$ years. Three participants had psychotherapist training additional to the BSW degree. The analysis resulted in two categories with four subcategories (Table 1). The categories and subcategories are presented in Table 1 and illustrated with verbatim quotations from the interviews.

\section{Knowledge in Practice}

The participants explained that they acquired their knowledge of interventions from different methods and theories, describing their knowledge as eclectic and piecemeal. They were not always aware of what method or which theory they were applying in their daily clinical practice.

\section{Practice Based on Research Evidence or Experience}

Knowledge and skills that were based on research evidence or theory were described as attributes that increased a social worker's confidence in dealing with patients, whereas the lack of these attributes could lead to stress, emotional strain and feelings of insecurity.

If there are no complications, I feel fairly confident with what I am doing, but as soon as things get more complex, it's important to know clearly what theories to lean on or methods to apply. Otherwise, it can be very stressful and lead to self-doubt when not knowing how to support the patient. (Focus group 1)

Experience-based practice was described as equally important as practice based on research evidence, although the

Table 1 An illustration of categories and subcategories

\begin{tabular}{ll}
\hline Categories & Subcategories \\
\hline Knowledge in practice & $\begin{array}{c}\text { Practice based on research evidence } \\
\text { or experience } \\
\text { Obtaining new evidence of practice }\end{array}$ \\
$\begin{array}{l}\text { Challenges in relation to the } \\
\text { implementation of EBP }\end{array}$ & $\begin{array}{l}\text { Research and the social work context } \\
\text { Barriers and facilitating factors }\end{array}$ \\
\hline
\end{tabular}


participants perceived that the status of the latter, as discerned by society and their managers, was much higher compared with experience-based practice. Another important aspect of knowledge in daily clinical practice was the patients' participation and information regarding their situation.

\section{Obtaining New Evidence of Practice}

The participants' main sources of new knowledge were their colleagues, supervisors, mass media, the hospital's intranet and different short courses. All the participants took part in regular supervision sessions (i.e. $2-4 \mathrm{~h}$ once or twice every month) in which they were observed by a formally trained supervisor. Most of the participants turned to a colleague for advice and support when they were unsure of how to handle a situation. They also expressed a wish for more consultation with colleagues, i.e. observe/attend a colleague's patient counseling to gain new perspectives. However, most of the participants were uncomfortable with the idea of a colleague observing/attending one of their own patient counseling, arguing that they would rather consult than be consulted. Team discussions with colleagues from different professions were also described as events that often provided new knowledge and insights. Turning to a physician in cancer or palliative care or contacting a county council lawyer for legal advice were other examples of collegial support. The hospital's intranet was sometimes used to locate specific manuals and guidelines. The participants expressed interest in using research evidence in intervention and as support to translate research into practice. However, many of the participants felt that research about their specific work area was lacking. They sometimes consulted members of the staff at the hospital library to obtain research studies and information. The participants described the Internet, radio and newspapers as common sources of knowledge on specific topics related to their daily work. Homepages on the Internet and scientific radio/ TV programs were considered reliable sources information. In addition, the participants discussed the importance of established national guidelines, protocols and standards to assist in the care process.

I can write a keyword on the Internet and then wait and see what I get from that... Then I listen to the radio a lot. For example, the program "Body and Soul" and "Science program" and all that stuff... Then there is the National Board of Health and Welfare with their national guidelines and such... that is also useful. (Focus group 4)

\section{Challenges in Relation to the Implementation of EBP}

\section{Research and the Social Work Context}

The participants' general perception was that there was little research on medical social work. In addition, most of the participants believed that research was often based on randomized controlled trials, which they did not perceive as being relevant to the complexities and variabilities that occur in their daily work. They also expressed an uneasy feeling of discomfort or fear of being scrutinized and judged by researchers or others in different professions who did not fully understand the context of their clinical practice. They also had mixed feelings about research and its applicability within the context of medical social work. Criticisms of EBP included the notion that work in accordance with EBP was demanding, that it tended to be based on natural science models to investigate social phenomena and was sometimes seen as a threat to person-centeredness, i.e. it did not consider the ever-changing complexity of the social work context.

Concerning evidence, when I started working in this field, no one talked about evidence. Now that was about 25 years ago, so it's been a while. But in recent years EBP has become popular. The need to explain and connect everything... Social work has always been more practical, not so much in terms of theoretical concepts but more social work. You know, it's what we do- practical work. (Focus group 3)

On the other hand, EBP was thought to include the patient's preferences as well as research and professional expertise. Most participants reported that they were not sure to what degree, if at all, their work was evidence-based.

"Evidence-based practice feels like just a requirement. Although we do use...well... proven experience, right?" (Focus group 5)

\section{Barriers and Facilitating Factors}

The participants reported the lack of opportunities for recurrent, structured discussions involving the exchange of knowledge between colleagues as being a barrier to transform individual knowledge into more general knowledge. For example, if someone had been to a conference or lecture, there was no protocol for the dissemination of the information acquired at the conference.

When someone has been at a conference or attended a course, it's important that we share that information at our regular workplace meetings. This is one suggestion on how to share and make use of newly obtained 
knowledge. I mean, there's a big difference whether one has worked for 1 year or if one has worked for many years, and depending on which continuing education and courses one has been taking... My knowledge is not only mine, but it needs to become 'our' knowledge. (Focus group 2)

The participants felt that the manager had the influence to facilitate or thwart the implementation of new knowledge. They also explained that they did not know how to evaluate new knowledge, such as research results, or how to integrate any newly acquired research evidence into their clinical practice. The participants expressed a need for a more positive environment and greater support from the manager in relation to the prioritization and structuring of time, as this would provide the time required to find new information, manuals and guidelines. The participants expressed a desire to appropriate time earmarked for reflection on how new research evidence could be integrated into practice.

I wish that our manager and the organizational management took some responsibility. We are busy with our everyday work, but as a manager you should lead the development forward and the manager needs to be at the forefront, obtaining new knowledge and perhaps discussing and highlighting these issues on research and so on... The pursuit of these issues, as well as the development of a sense of enthusiasm and inspiration among the staff is included in the manager's area of responsibility... (Focus group 1)

Searching and reading theoretical and empirical studies were seen as time consuming. The participants indicated that there is considerable difficulty involved in the transformation of information contained in a study into actual procedures that could be put into practice. The participants expressed the desire for knowledgeable people to provide relevant research and assist in the application of that research. In addition, they explained that although they wanted more research on medical social work, they stated that they were unable to take part in studies because they were not accustomed to research activities. They indicated a willingness to be a part of a research endeavor, but seemed to feel that they lacked the time (and skills and knowledge) required to do so.

\section{Discussion}

In this study, medical social workers' perceptions of EBP imply challenges and complexities involving not only their interpretation of different concepts (i.e. EBP) but also their values of the concepts. Implementation of EBP is likely to be unsuccessful if the medical social workers feel unmotivated to adapt to new routines or to perform new interventions, and instead feel forced into a top-down process in which they perceive that they are not taken seriously. An emphasis on quantitative studies that do not address the type of interpersonal ethical dilemmas that the medical social workers face, might lead to EBP being seen as too narrow. Because the participants emphasized a more 'subjective' perspective (e.g., the patient's individual situation and the value of their individual professional experience), it needs to be acknowledged that these are already important parts of EBP. According to the iPARIHS framework, the outcome of implementation results from the interplay between the three core constructs: recipients, context and innovation (Harvey and Kitson 2015). The perception that EBP, as articulated by the medical social workers in this study, is diametrically different from experience-based knowledge and practice is important knowledge. In this study, the medical social workers' defined themselves as users of experience-based knowledge and descriptions imply that they perceive research as too positivistic, making it difficult to adapt such research across individual situations or contexts.

There is a need to acknowledge that context, patient preferences and professional expertise (of which experiencebased expertise is a part) are considered as equally important components (cf. Sacket et al. 1996). According to the present results, these components are already applied in the participants' everyday work. The participants' perception of the EBP concept underlines its complexity in relation to the social work profession. In general, social work education and training often lack or fail to incorporate research evidence (Bledsoe et al. 2007; Mullen and Bacon 2006), which may be one explanation for their skepticism towards EBP, i.e. not being so familiar to the concept. Moreover, the social work settings are multiple (e.g., community work, child care and medical social), which implies challenges as how to translate research evidence into each unique social work setting. The medical social workers in our study had a BSW degree, and perhaps it is possible that further education and training could constitute the necessary support to ensure a more refined EBP concept relative to what they already apply in their daily work. Several studies have proposed that social work in general demands a different view of knowledge than traditional EBP, largely because social work is often complex and un-predictable and therefore difficult to standardize (Gray and McDonald 2006; Blom 2009). The present findings seem to suggest that social workers within a healthcare context are caught between two scientific conceptualizations. Medical social workers have been educated within the field of social science, which is mostly epistemologically based in social constructivism and psychodynamic perspectives. Professionally, however, they are developing within a medical healthcare context that is generally positivistic (i.e. objective, linear, predictive and expert-based). If the medical social workers were unfamiliar with the EBP 
concept and lack the guidance and support to integrate a social constructivist perspective with a positivistic perspective in practice, they may find it difficult, if not impossible, to implement EBP.

Although the participants expressed some skepticism towards research evidence, they still applied EBP in relation to the inclusion of patients' preferences and the clinical expertise of the practitioners. Thus, the skepticism may have been derived from a misinterpretation of the EBP concept. Searching for best research evidence and then evaluating the evidence were the two elements the participants considered most difficult and complicated. Different barriers, such as time, lack of support and difficulties translating research into viable practical methods, were expressed by the participants. Lack of time was found to be an important barrier to the implementation of EBP. Time has also previously been shown to be a barrier to the use of EBP among healthcare professionals (Carlson and Plonczynski 2008; Heiwe et al. 2011). Insufficient time may constitute a major source of work-related stress if heavy demands are placed on the medical social worker without providing additional time and resources. Restricted time may also prevent or limit medical social workers the possibility to support one another in consultations and daily dialogue. Therefore, the perceived time issue described in this study must be considered when incorporating EBP into daily clinical practice. Manuel et al. (2009) emphasized the need to consider the real-world clinical complexities (e.g., context and culture) when implementing EBP. One of their suggestions for successful EBP implementation in social work settings is to apply a multilevel approach that would include increased collaboration (through partnership) with different organizations.

The participants in our study often mentioned contextual factors when discussing EBP and tended to have the perception that their field is contextually unique. This perception can be seen in the comments the participants made regarding consultation, implementation routines, barriers for implementation and managerial support. In this study, the manager was identified as a central member in the process of knowledge exchange and EBP. For example, the manager is expected to present and initiate a clear strategy on how to prioritize time for this process and how to translate research results into a specific context. The need for the organization to provide more time (and decrease constraints) to assess, evaluate and assimilate research the literature has previously been suggested as a way to facilitate EBP (Stewart et al. 2012; Wharton and Bolland 2012). The participants suggested that the manager should support the implementation of new practices. The manager's role is a well-known contextual factor in different implementation theories. It is generally agreed that managers have a strategic role in research transfer (Gifford et al. 2007), but the key supporting leadership behaviors are yet to be synthesized and leadership interventions need evaluation and validation (Gifford et al. 2014; Sandström et al. 2011).

Facilitation is a known active component for successful implementation. In this study, the participants identified several factors that could contribute to the enhancement of EBP. For example, they suggested that a special person (facilitator) should be responsible for supporting EBP by providing relevant research studies and helping translate research results into a specific context. The participants also wanted a clearer structure, help in prioritizing time and to be given organizational support and encouragement from management relative to conducting structured knowledge dialogues with colleagues and when performing consultations. Because some of the participants were more familiar with EBP than others, those with more familiarity could be regarded as facilitators. As facilitators, they could lead the discussions or consultations with colleagues less familiar with EBP. The participants' suggestions for knowledge enhancement (e.g., the process by which individual knowledge is transformed into general knowledge through a structured process of collegial dialogues) could constitute a part of the EBP implementation process. The exchange of knowledge among colleagues through consultation and structured (recurrent) information-sharing platforms, together with managerial support and encouragement, as well as the addition of a person whose primary duty is to assist the participants in the process of research translation, would represent a more bottom-up process. These practice-based suggestions could be considered in EBP implementation within the context of medical social work.

\section{Study Limitations}

The study has some limitations. First, the focus group interviews were conducted by different data collectors that led to some differences in the focus of the interviews. The analysis, however, concentrated only on the data related to the aim of the study. Second, because the study focuses on medical social workers within a hospital setting, the findings may not be applicable for social workers within other contexts. Third, the study was conducted in a European setting, which may imply some differences compared with other global areas (e.g., the USA). However, the primary aim of this qualitative study is not to generalize the findings, but rather it is up to each reader to consider the findings in relation to his or her specific context.

\section{Conclusions}

This study explores medical social workers' perceptions of implementing EBP. Our results show that medical social workers tend to interpret research evidence as theoretical and 
positivistic while perceiving their own knowledge as eclectic and experience-based. However, our findings also suggest that medical social workers do express some interest in research findings, but need support to translate research into practice. The participants' suggestion for facilitating factors needs further investigation. A specially designated person responsible for supporting the increased use of research findings and who supports prioritization of time, the sharing of knowledge and who encourages more consultations should be prioritized. All these factors need to be considered when promoting the implementation of EBP within medical social work settings. Furthermore, a holistic and person-centered perspective, in all its complexity, requires a joint effort from different healthcare professionals based on different kinds of knowledge sources. Results from this study illustrate the need for interprofessional dialogue about EBP and its implementation in practice, with a common focus on patient outcomes.

Acknowledgements We are grateful to the medical social workers who participated in this study.

\section{Compliance with ethical standards}

Conflict of interest The authors declare that they have no conflict of interest.

Open Access This article is distributed under the terms of the Creative Commons Attribution 4.0 International License (http://creativecommons.org/licenses/by/4.0/), which permits unrestricted use, distribution, and reproduction in any medium, provided you give appropriate credit to the original author(s) and the source, provide a link to the Creative Commons license, and indicate if changes were made.

\section{References}

Bellamy, J. L., Bledsoe, S. E., \& Traube, D. E. (2006). The current state of evidence-based practice in social work: A review of the literature and qualitative analysis of expert interviews. Journal of Evidence Based Social Work, 3(1), 23-48. https://doi.org/10.1300/ J394v03n01_02.

Bergmark, A., \& Lundström, T. (2011). Guided or independent? Social workers, central bureaucracy and evidence-based practice. European Journal of Social Work, 14(3), 323-337. https://doi. org/10.1080/13691451003744325.

Björkenheim, J. (2007). Knowledge and social work in health care-the case of Finland. Social Work in Health Care, 44(3), 261-278. https://doi.org/10.1300/J010v44n03_09.

Bledsoe, S. E., Weissman, M. M., Mullen, E. J., Ponniah, K., Gameroff, M., Verdeli, H., Mufson, L., Fitterling, H., \& Wickramaratne, P. (2007). Empirically supported psychotherapy in social work training programs: Does the definition of evidence matter? Research on Social Work Practice, 17, 449-455. https:// doi.org/10.1177/1049731506299014.

Blom, B. (2009). Knowing or un-knowing? That is the question. In the era of evidence-based social work. Practice Journal of Social Work, 9(2), 158-177. https://doi.org/10.1177/1468017308101820.
Carlson, C. L., \& Plonczynski, D. J. (2008). Has the barriers scale changed nursing practice? An integrative review. Journal of Advanced Nursing, 63(4), 322-333. https://doi. org/10.1111/j.1365-2648.2008.04705.x.

Fugl-Meyer, K. S. (2016). A medical social work perspective on rehabilitation. Journal of Rehabilitation Medicine, 48(9), 758-763. https://doi.org/10.2340/16501977-2146.

Gifford, W., Davies, B., Edwards, N., Griffin, P., \& Lybanon, V. (2007). Managerial leadership for nurses' use of research evidence: An integrative review of the literature. Worldviews on Evidence-Based Nursing, 4(3), 126-145. https://doi. org/10.1111/j.1741-6787.2007.00095.x.

Gifford, W. A., Holyoke, P., Squires, J. E., Angus, D., Brosseau, L., Egan, M., Graham, I. D., Miller, C., \& Wallin, L. (2014). Managerial leadership for research use in nursing and allied health care professions: A narrative synthesis protocol. Systematic Reviews, 3(1), 57. https://doi.org/10.1186/2046-4053-3-57.

Gray, M., \& McDonald, C. (2006). Pursuing good practice? The limits of evidence-based practice. Journal of Social Work, 6(1), 7-20. https://doi.org/10.1177/1468017306062209.

Harvey, G., \& Kitson, A. (2015). Implementing evidence-based practice in healthcare: A facilitation guide. Abington: Routledge.

Haynes, R. B., Devereaux, P., \& Guyatt, G. H. (2002). Clinical expertise in the era of evidence-based medicine and patient choice. ACP Journal Club, 136(2), A11-A14. https://doi.org/10.1136/ ebm.7.2.36.

Heiwe, S., Kajermo, K. N., Tyni-Lenné, R., Guidetti, S., Samuelsson, M., Andersson, I. L., \& Wengström, Y. (2011). Evidence-based practice: Attitudes, knowledge and behaviour among allied health care professionals. International Journal for Quality in Health care, 23(2), 198-209. https://doi.org/10.1093/intqhe/ mzq083.

Heiwe, S., Nilsson-Kajermo, K., Olsson, M., Gåfvels, C., Larsson, K. \& Wengström, Y. (2013). Evidence-based practice among Swedish medical social workers. Social Work in Health Care, 52(10), 947-958. https://doi.org/10.1080/00981389.2013.834029.

Krippendorff, K. (2013). Content analysis: An introduction to its methodology (3rd edn.). London: Sage Publications.

Kvale, S. (2006). Interviews: An introduction to qualitative research interviewing. London: Sage Publications.

Manuel, J., Mullen, E. J., Fang, L., Bellamy, J., \& Bledsoe, S. E. (2009). Preparing social work practitioners to use evidence-based practice: A comparison of experiences from an implementation project. Research on Social Work Practice, 19(5), 613-627. https://doi.org/10.1177/1049731509335547.

McNeece, A., \& Thyer, B. A. (2004). Evidence-based practice and social work. Journal of Evidence-Based Social Work, 1(1), 7-25. https://doi.org/10.1300/J394v01n01_02.

Melnyk, B. M., \& Gallagher-Ford, L. (2015). Implementing the new essential evidence-based practice competencies in real-world clinical and academic settings: Moving from evidence to action in improving healthcare quality and patient outcomes. Worldviews on Evidence-based Nursing, 12(2), 67-69. https://doi.org/10.1111/ wvn. 12089.

Morago, P. (2006). Evidence-based practice: from medicine to social work. European Journal of Social Work, 9(4), 461-477. https:// doi.org/10.1080/13691450600958510.

Morgan, D. L. (1997). Focus groups as qualitative research (2nd edn.). London: Sage Publications.

Mullen, E. J., \& Bacon, W. (2006). Implementing of practice guidelines and evidence-based treatment: A survey of psychiatrists, psychologists and social workers. In A. R. Roberts \& K. R. Yeager (Eds.), Foundations of evidence-based social work practice (pp. 81-92). New York, NY: Oxford University Press. 
Petr, C. G., \& Walter, U. M. (2009). Evidence-based practice: A critical reflection. European Journal of Social Work, 12(2), 221-232. https://doi.org/10.1080/13691450802567523.

Rubin, A., \& Parrish, D. (2007). Views of evidence-based practice among faculty in master of social work programs: A national survey. Research on Social Work Practice, 17(1), 110-122. https:// doi.org/10.1177/1049731506293059.

Rycroft-Malone, J. (2004). The PARIHS framework-a framework for guiding the implementation of evidence-based practice. Journal of Nursing Care Quality, 19(4), 297-304. https://doi. org/10.1097/00001786-200410000-00002.

Sackett, D. L., Rosenberg, W. M., Gray, J. A., Haynes, R. B., \& Richardson, W. S. (1996). Evidence based medicine: What it is and what it isn't. BMJ, 312, 71-72. https://doi.org/10.1136/ bmj.312.7023.71.

Sandström, B., Borglin, G., Nilsson, R., \& Willman, A. (2011). Promoting the implementation of evidence-based practice: A literature review focusing on the role of nursing leadership. Worldviews on Evidence-Based Nursing, 8(4), 212-223. https://doi. org/10.1111/j.1741-6787.2011.00216.x.

Satterfield, J. M., Spring, B., Brownson, R. C., Mullen, E. J., Newhouse, R. P., Walker, B. B., \& Whitlock, E. P. (2009).
Toward a transdisciplinary model of evidence-based practice. The Milbank Quarterly, 87(2), 368-390. https://doi. org/10.1111/j.1468-0009.2009.00561.x.

Squires, J. E., Estabrooks, C. A., Gustavsson, P., \& Wallin, L. (2011). Individual determinants of research utilization by nurses: A systematic review update. Implementation Science, 6(1), 1. https:// doi.org/10.1186/1748-5908-6-1.

Stewart, R. E., Chambless, D. L., \& Baron, J. (2012). Theoretical and practical barriers to practitioners' willingness to seek training in empirically supported treatments. Journal of Clinical Psychology, 68(1), 8-23. https://doi.org/10.1002/jclp.20832.

Wharton, T. C., \& Bolland, K. A. (2012). Practitioner perspectives of evidence-based practice. Families in Society, 93(3), 157-164. https://doi.org/10.1606/1044-3894.4220.

Wike, T. L., Bledsoe, S. E., Manuel, J. I., Despard, M., Johnson, L. V., Bellamy, J. L., \& Killian-Farrell, C. (2014). Evidence-based practice in social work: Challenges and opportunities for clinicians and organizations. Clinical Social Work Journal, 42(2), 161-170. https://doi.org/10.1007/s10615-014-0492-3. 